\title{
FutureJournal
}

FUTURE STUDIES RESEARCH JOURNAL:

TRENDS AND STRATEGIES

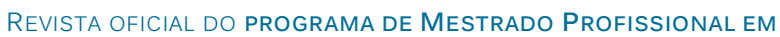

Gestão de Negócios do Profuturo - Programa de Estudos

DO FUTURO - DA FIA BUSINESS SCHOOL

Editora Científica: Renata Giovinazzo Spers

Editor Internacional: James Terence Coulter Wright

Editor de Relato Técnico: Daniel Estima de Carvalho

Avaliação: Double Blind Review, pelo SEER/OJS

Revisão: Gramática, normativa e de layout

Recebido em: 01/12/2017 Aprovado em: 25/04/2018

\section{Volatilidade e Transmissão dos Preços Internacionais do Trigo para os Preços Domésticos do Trigo e Derivados no Brasil}

\author{
Jessie Divina Silva Rezende \\ Universidade Federal de Uberlândia (UFU), Brasil \\ jessiedrezende@hotmail.com \\ Odilon José de Oliveira Neto \\ Universidade Federal de Uberlândia (UFU), Brasil \\ professorodilon@yahoo.com.br \\ Kelly Aparecida Silva \\ Universidade Federal de Uberlândia (UFU), Brasil \\ kelly_asilva@yahoo.com.br
}

\section{RESUMO}

O Brasil ocupa a terceira posição no ranking de importação de trigo e tem esse grão como o segundo maior produto em participação na pauta geral de importações. Assim, torna ainda mais importante a atenção quanto à volatilidade do preço internacional do trigo, a fim de compreender melhor o comportamento e a transmissibilidade do preço para um planejamento mais eficiente dos agentes na cadeia produtiva. Nesse sentido, este estudo tem por objetivo analisar a volatilidade e a transmissão do preço do trigo internacional para os preços domésticos desse grão e seus derivados no Brasil. Para atingir o objetivo, utilizaram-se testes estatísticos, como estatística descritiva, teste de raiz unitária de ADF, teste de cointegração e aplicação do modelo VEC. Como resultado, verificou-se uma correlação forte e positiva do preço do trigo brasileiro com os preços do trigo norte-americano $(0,92)$, e moderada e positiva com os preços do trigo argentino $(0,68)$. No curto prazo, o modelo VEC indicou que uma hipotética variação de $1 \%$ no preço do trigo argentino levaria a um aumento de $1,34 \%$, no preço do trigo brasileiro, e a variação de $1 \%$ no preço do trigo norte-americano, ampliaria em 1,29\% a variação do preço da farinha de trigo no Brasil. Em suma, concluiu-se que são fortes as evidências de transmissão dos preços internacionais do trigo para os preços domésticos do trigo e derivados no Brasil. 
PALAVRAS CHAVE: Trigo. Transmissão de preços. Volatilidade de preços.

\title{
Volatility and Transmission of International Prices of Wheat for Domestic Wheat and Derivative Prices in Brazil
}

\begin{abstract}
ABSTACT
Brazil occupies the third position in the wheat import rank and has this grain as the second biggest item in the general imports issue. Thus, becomes even more important the attention regarding the volatility of the wheat's international price, in order to have a better comprehension of the price behavior and transmissibility for a more efficient planning by the agents in the productive chain. Seen in these terms, this study has as a purpose to analyze the volatility and the transmission of the wheat's international price to the domestic prices of this grain and its by-products in Brazil. To reach this target, it was used statistics tests, such as descriptive statistics, ADF unitary root test, cointegration test and application of the VEC model. As a result, it was verified a strong and positive correlation of the price of Brazilian wheat with North American one (0.92), and a moderate and positive compared with the Argentinean wheat (0.68). In the short term, the VEC model indicated that a hypothetical variation of $1 \%$ in the Argentinean wheat will led to an increase of $1.34 \%$ in the price of the Brazilian one, and a $1 \%$ price variation in the North American wheat will increase in $1.29 \%$ the price of the wheat flour in Brazil. All in all, is concluded there are strong evidences of transmission in the wheat's international prices to the domestic ones and its by-products in Brazil.
\end{abstract}

KEY-WORDS: Wheat. Price transmission. Price volatility 


\section{INTRODUÇÃO}

O trigo tem um peso significativo para a economia agrícola global, já que é o segundo cereal mais produzido e consumido em âmbito mundial, atrás apenas do milho. Além de ser um grão com demanda concentrada nos países em desenvolvimento, tendo em vista o fato da população utilizar expressivamente esse grão na fabricação de derivados voltados à alimentação. O trigo, assim como o arroz, é um alimento básico da população em muitos países com baixa e média renda per capita, de acordo com informações divulgadas pelo United States Department of Agriculture [USDA], (2013).

No Brasil, a produção de trigo (ou cultura tritícola) concentra-se nas regiões Sul, Sudeste e Centro-Oeste, motivada, principalmente, pelas condições climáticas favoráveis. Entretanto, a produção nacional não é suficiente para atender à demanda interna, resultando na necessidade de importação dessa commodity. De acordo com o Ministério da Agricultura, Pecuária e Abastecimento [MAPA], (2016) somente 11 países estavam autorizados a exportarem trigo para o Brasil, destacando-se, dentre eles, a Argentina e os Estados Unidos da América (EUA), os quais estão entre os cinco maiores produtores e exportadores mundiais de trigo.

Como os principais países em volume de exportação do trigo para o Brasil são, respectivamente, a Argentina e os EUA, uma análise temporal da volatilidade e a transmissão dos preços para o Brasil é importante, uma vez que oscilações do preço nesses mercados podem afetar as expectativas em relação aos resultados futuros da indústria brasileira e até mesmo atingir o consumidor final.

No que tange ao mercado, é importante ressaltar que o Brasil ocupa a terceira posição no ranking de importação de trigo (atrás apenas do Egito e da Indonésia), conforme dados de USDA (2016). Vale destacar também que o trigo é o segundo item de maior participação na pauta de importações brasileiras, o que reforça sua posição como um dos maiores importadores dessa commodity agrícola.

O consumo nacional de trigo manteve-se, desde 2006, em torno de 10 milhões de t/ano, ao passo que a produção interna oscilou em torno de 5 e 6 milhões de t/ano, conforme relatam Jesus, Sidonio e Moraes (2011). De acordo com dados da Associação Brasileira de Indústria do Trigo [ABITRIGO], (2016) o Brasil importa cerca de cinco milhões de t/ano, atingindo o nível máximo de 
importação no ano de 2013, com 7.273.270,75 toneladas de trigo, o que corresponde a 332,01 US\$/t.. Mas, segundo estimativas do USDA (2016), a importação brasileira de trigo deve atingir, aproximadamente, 7,7 milhões de toneladas em 2024/25 (ABITRIGO, 2016).

Ao considerar a grande quantidade de trigo importado pelo Brasil, tornase ainda mais importante a atenção quanto à volatilidade do preço internacional a fim de melhor compreender o comportamento $e$ transmissibilidade do preço com vistas a um planejamento mais eficiente dos agentes na cadeia produtiva. Nesse sentido, Brown (2002) destaca a volatilidade dos preços nas commodities como um problema para os países em desenvolvimento, principalmente, por essa volatilidade afetar a estabilidade econômica da indústria.

Segundo a Coopération Internationale pour le Développementet la Solidarity [CIDSE], (2011) o grau de impacto da volatilidade do preço agrícola em países em desenvolvimento depende da transmissão dos mercados globais para os locais, visto que os países que já enfrentam dificuldades serão ainda mais pressionados, o que pode, em momentos de pico, afetar não somente a indústria, mas também outros agentes da cadeia produtiva.

Diante do exposto, a presente pesquisa tem como intuito responder a seguinte problemática: A volatilidade do preço do trigo nos principais mercados internacionais é transmitida aos preços do trigo e derivados no mercado interno brasileiro? Quanto a esse questionamento, ressalta-se que a análise do processo de transmissão de preço e da volatilidade parte da hipótese de que a variação nos preços dos principais mercados produtores do trigo é transmitida aos preços praticados no Brasil.

A relevância deste estudo parte, principalmente, da baixa produção nacional de trigo, fato que coloca o país em um cenário de dependência de importação da referida commodity. Além disso, o trigo se destaca como uma das principais matérias-primas para a indústria alimentícia brasileira, sendo utilizado, principalmente, na produção: de farinha (componente da cesta básica brasileira), de pães, massas, bolos, biscoitos e similares.

Ademais, espera-se que o presente estudo contribua com informações relevantes para a tomada de decisões dos integrantes do setor tritícola, em especial, para os produtores de derivados do trigo para melhorar a compreensão acerca de informações sobre possíveis impactos da dinâmica dos 
preços no consumidor brasileiro. Além disso, o intento é contribuir com informações para a gestão de políticas públicas e privadas de incentivo e intensificação da produção nacional. A partir das informações geradas pela presente pesquisa, espera-se ampliar a discussão acadêmico-cientifica em torno do tema volatilidade e transmissão de preços agrícolas, principalmente, considerando a cultura tritícola.

Sendo assim, o objetivo deste trabalho é analisar a volatilidade e a transmissão do preço do trigo internacional para os preços domésticos do trigo e derivados no Brasil. O período do estudo encontra-se compreendido entre fevereiro de 2004 e fevereiro de 2017, em razão da disponibilidade temporal de dados históricos do preço do trigo no país. O artigo encontra-se estruturado em cinco seções, sendo esta (1) a seção introdutória seguida, respectivamente, pela (2) fundamentação teórica, (3) procedimentos metodológicos, (4) resultados e discussões e (5) considerações finais.

\section{REVISÃO DE LITERATURA}

Esta seção tem por finalidade fundamentar teoricamente, além de oferecer maior entendimento acerca da volatilidade e transmissão de preços das commodities agrícolas. Para tanto, são apresentados e discutidos estudos publicados em periódicos científicos nacionais e internacionais que abordam o assunto, tratando dos diferentes segmentos agrícolas, dentre eles, os mercados de soja, trigo, algodão, café arábica, tomate, alface crespa e macaxeira, setor sucroalcooleiro, fumo e arroz.

A princípio Mundlak e Larson (1992) realizaram um estudo com o intento de responder duas perguntas básicas que relacionam os preços internos e os preços mundiais das commodities agrícolas: (1) as variações dos preços mundiais são transmitidas para os preços domésticos? (2) variações, nos preços mundiais, constituem um componente importante nas variações de preços no mercado interno? Para essa análise, foram utilizados dados de 58 países, levando em consideração uma abrangência de dez anos (1968 a 1978). Os referidos autores consideraram também dados da Comunidade Europeia, porém, nesse caso, o período foi de 1961 até 1985. Nessa análise, os autores 
constataram que, na maioria das vezes, as variações dos preços mundiais das commodities agrícolas são transmitidas para os preços nos mercados internos.

Um dos primeiros estudos brasileiros sobre transmissão de preços foi o dos autores Margarido, Kato e Ueno (1994), os quais tiveram o intuito de analisar a relação entre os preços de tomate no atacado e no varejo. Tendo como referência o Estado de São Paulo, verificou-se que a transmissão do preço foi parcialmente instantânea e que o restante é transmitido no mês seguinte. Para essa análise, foram utilizados dados com uma série temporal mensal que compreende 22 de maio de 1970 a dezembro de 1992.

No que diz respeito ao mercado da soja em grão, destacam-se os estudos de autores como: Margarido, Turolla e Fernandes (2001), W. Silva, Santo e L. Silva (2003), Corso, Silva e Duclós (2006), Tonin e Barczsz (2007) e Silva e Machado (2009).

Margarido et al. (2001) analisaram a elasticidade da transmissão do preço da soja em grão, utilizando como referência o Porto de Rotterdam e o Brasil entre julho de 1994 e setembro de 2000. Para o estudo, foi utilizado o modelo de correção de erros, com e sem restrições, nos parâmetros baseado no modelo de Mundlack e Larson (1992). Os resultados mostraram que os preços da soja brasileira apresentam tendência de eliminar mais rapidamente, ou seja, no curto prazo, os desiquilíbrios transitórios de preços que o Porto de Rotterdam. Na perspectiva de longo prazo, foi verificado que a taxa de câmbio e os preços de Porto de Rotterdam são transmitidos para os preços brasileiros do grão de soja, sendo as bruscas oscilações de preços da soja causadas quando há variações na taxa de câmbio de Rotterdam, de modo que os preços vão aumentando até sete meses após o choque e, depois, ao longo do tempo, se estabilizam.

Tendo como referência os preços da soja americana e brasileira, W. Silva et al. (2003) verificaram as relações de cointegração (longo prazo) existentes entre os preços em valores nominais, considerando um período próximo ao determinado no estudo de Margarido et al. (2001). Com uma série temporal baseada em dados mensais, que vai de janeiro de 1995 a agosto de 2002, os autores utilizaram o método de cointegração proposto por Engle e Granger (1987) e encontraram um resultado semelhante ao verificado no estudo de Margarido et al. (2001), no qual é evidenciada a existência de relações de 
cointegração ou de equilíbrio de longo prazo entre os preços da soja no Brasil e nos EUA.

Corso et al. (2006), também realizaram estudo similar ao de W. Silva et al. (2003), porém seus dados de análise foram de janeiro de 1995 e se estendem até janeiro de 2005. Mesmo com uma série temporal mensal maior, os pesquisadores encontraram os mesmos resultados. Já Tonin e Barczsz (2007) realizaram seu estudo referente a região de Maringá (Paraná, Brasil), e a cotação da bolsa de valores nos EUA, levando em consideração a Lei do preço único, com uma série temporal de julho de 1994 a dezembro de 2007. Ao utilizar os testes de estacionariedade, de causalidade de Granger e de cointegração, os autores encontraram também uma relação de longo prazo, sugerindo que a bolsa internacional pode ser considerada uma boa referência para os preços domésticos de Maringá e vice-versa. Na mesma perspectiva e corroborando os estudos de transmissão de preço no mercado da soja, Silva e Machado (2009) encontraram que, embora, no longo prazo, os preços sejam cointegrados, no curto prazo, não há uma relação forte entre o mercado brasileiro e o norte-americano.

Com um objetivo um pouco diferente dos estudos anteriores, Margarido et al. (2014) analisaram o relacionamento tanto em termos de volatilidade, quanto de transmissão de preços entre os preços internacionais do petróleo e o grão de soja. Utilizando-se de uma série temporal entre janeiro de 1980 e outubro de 2010, e da aplicação dos testes ADF, de Causalidade de Granger (1969), de Cointegração de Johansen (1988), de Exogeneidade, de Modelo Vetorial de Correção de Erro (VEC), da Decomposição da Variância dos Erros de Previsão, da Função de Resposta de Impulso e também do modelo GARCH Multivariado, os pesquisadores encontraram resultados que indicaram que, no longo prazo, as variações nos preços internacionais do petróleo são timidamente transferidas para os preços da soja, por outro lado, há ausência de relacionamento entre as variáveis no curto prazo.

Ao considerar o setor tritícola, destacam-se os estudos como os Bessler, Yang e Wongcharupan (2003), Yang, Zhang e Leatham (2003), Margarido, Bueno, Martins, Tomaz (2007); Brummer, Cramon-Taubadel e Zorya (2009), Coronel, Amorim, Sousa, Lima (2010); Alves, Silva, Nascimento, Mascarenhas, Djau (2014) e Alves, Cardoso, Felipe, Campion (2015). 
Bessler et al. (2003) examinaram as relações dinâmicas dos preços do trigo em cinco países considerados grandes regiões produtoras, sendo eles: o Canadá, a União Europeia, a Argentina, a Austrália e os EUA. Os resultados empíricos mostraram que o Canadá e os EUA são líderes no preço do trigo entre esses mercados e que os EUA têm um efeito significativo sobre os preços nos outros três mercados, exceto no Canadá.

O estudo de Yang et al. (2003) analisou os preços futuros, a volatilidade e a transmissão de preços nos EUA, Canadá e União Europeia, que foram os três maiores produtores e exportadores de trigo entre 1996 até 2002. Nesse estudo, foi evidenciado que os preços na União Europeia são autodependentes e não sofrem influências nesse período, mas pode influenciar, no longo prazo, os preços dos EUA, o que contraria o estudo de Bessler et al. (2003). O Canadá, por sua vez, é muito influenciado pelos EUA quanto à transmissão de preço, mas não ocorre o inverso. Em relação à transmissão de volatilidade, ocorre do Canadá e da União Europeia para os EUA e da União Europeia para o Canadá, mas não ocorre o inverso em nenhuma das situações. Além disso, os autores apontam que não há um mercado com liderança distintiva e, sim, que todos apresentam, em certa medida, uma liderança, o que se configura como outra divergência com o estudo de Bessler et al. (2003) pois, neste os resultados apontaram que os EUA e o Canadá são líderes de mercado.

Com o apoio em testes estatísticos de raiz unitária, de causalidade de Granger, de cointegração de Johansen (1988), de estimação da função impulso-resposta, de decomposição da variância dos erros de previsão e estimação e da análise do modelo de correção de erros e teste de exogeneidade, Margarido et al. (2007) analisaram a elasticidade de transmissão de preços da farinha de trigo em São Paulo, bem como a taxa de câmbio e a cotação internacional do trigo em grão. Os autores adotaram, como base, a lei do preço único, abrangendo o período entre janeiro de 1999 e dezembro de 2005. Os resultados mostraram que, no longo prazo, as variações das cotações internacionais do trigo em grão e da taxa de câmbio são plenamente transmitidas para os preços da farinha de trigo na cidade de São Paulo.

No que tange ao mercado tritícola internacional, Coronel et al. (2010) verificaram a existência de uma relação entre o mercado argentino e o mercado internacional no que se refere aos preços do trigo, no período de 
janeiro de 1994 a abril de 2009. Foram utilizados, neste estudo, os testes de causalidade, de cointegração e o modelo de correção de erros. Os resultados sugerem que os preços internacionais influenciam nos preços argentinos, ou seja, as variações nos preços internacionais foram transmitidas para o mercado interno do trigo argentino.

Por sua vez, Alves et al. (2014), ao analisarem a relação dos preços do trigo em dois mercados regionais brasileiros e dois mercados internacionais, auferiram o grau de convergência entre os mesmos, no período de janeiro de 2004 a junho de 2012, seguindo a metodologia de Busetti et al. (2006). O resultado apresentou elevado grau de convergência entre os preços do trigo para os preços da Argentina e de Porto Alegre.

Com a intenção de analisar as relações de causalidade e de transmissão de preços da raiz de mandioca, da fécula de mandioca, da farinha de mandioca, do trigo, da farinha de trigo e do milho no Estado do Paraná, Alves et al. (2015) analisaram dados mensais entre janeiro de 1995 a dezembro de 2005. Nessa pesquisa, os autores adotaram o teste de causalidade de Granger e concluíram, como nos estudos de Coronel et al. (2010) e Alves et al. (2014), pela existência de uma forte relação de longo prazo entre trigo e farinha de trigo.

Estudos sobre transmissão de preços também foram realizados com commodities de mercados mais restritos ou que apresentam particularidades quanto aos mecanismos de negociação. Dentre esses trabalhos, encontram-se estudos sobre as commodities algodão, café arábica, alface crespa e macaxeira, açúcar e álcool, fumo e arroz.

Ao analisar a elasticidade de transmissão de preços no mercado de algodão brasileiro, a partir de dados mensais entre janeiro de 1985 e dezembro de 2000, Barbosa, Margarido e Nogueira (2002), ao contrário de estudos anteriores, apontaram que, em relação ao algodão brasileiro, não é válida a Lei do Preço Único pelo fato de não haver uma transmissão total de preços em relação aos preços internacionais para os preços domésticos no Brasil. Ao utilizar o teste de exogeneidade, os pesquisadores estimaram que os preços internos brasileiros não reagem às mudanças em uma relação de longo prazo. Infere-se que esse resultado possa estar associado às intervenções governamentais, entre 1985 e 1988, e também às condições favoráveis de financiamentos no setor agrícola. 
Com o propósito de analisar a integração espacial de preços nos principais estados brasileiros produtores de café arábica, quais sejam, São Paulo e Minas Gerais, Nogueira, Aguiar e Lima (2005) concluíram que esses mercados são integrados. Para essa análise, os autores utilizaram o teste de raiz unitária, adotando o modelo estatístico de Dickey e Fuller Aumentado, o teste de cointegração, o modelo de correção de erros e o teste de causalidade. Como resultado, esses autores encontraram que a região do Cerrado de Minas tem reação de causa e influência nos preços das demais regiões, o que contrariou a expectativa de que seria, nesse caso, a região Sul de Minas Gerais, por se tratar de uma das maiores produtoras e exportadoras de café arábica do Brasil.

Silva e Lages (2011), com uma abordagem um pouco distinta dos autores citados anteriormente, analisaram produtos locais no Estado de Alagoas. Esse estudo teve como objetivo analisar a transmissão de preço da alface crespa e da macaxeira (aipim), tendo sido identificado que há certa dificuldade para o produtor repassar seus preços para o comércio varejista, o que pode ser associado à perecibilidade do produto.

Já no setor sucroalcooleiro, o estudo realizado por Block, Coronel e Veloso (2012) teve como objetivo analisar o processo de transmissão de preço, utilizando-se séries de preços de cana-de-açúcar, etanol hidratado e açúcar no Estado de São Paulo, no período de fevereiro de 1999 até setembro de 2010. A partir do teste vetorial de Autorregressão (VAR), encontraram que o preço desses produtos tem forte ligação e que o etanol hidratado influência nos demais, mas não sofre qualquer influência dos mesmos.

A partir de uma série temporal mensal, abrangendo dez anos, com início em janeiro de 1999 e término em dezembro de 2009, Freitas, Lírio e Coronel (2012) verificaram o mercado de fumo nacional (Brasil) e internacional quanto à sua magnitude na transmissão de preços e condições de integração. Os resultados apontaram para uma relação de equilíbrio de longo prazo e sensibilidade do preço nacional em relação aos preços internacionais. Além disso, encontrou-se que os preços recebidos pelo produtor no Paraná são sensíveis aos preços praticados no Rio Grande do Sul e em Santa Catarina. Santos, Wolff e Souza (2013) analisaram a transmissão dos preços do Rio Grande do Sul, Mato Grosso e Santa Catarina, principais estados produtores de arroz no país. Utilizando a metodologia dos modelos de vetores 
autorregressivos e também os modelos de correção de erros, de teste de cointegração, de causalidade, de decomposição de Cholesky, os autores chegaram à conclusão de que o preço do arroz, no Estado do Rio Grande do Sul, influência nos preços do estado de Santa Catarina e no volume armazenado em estoques públicos, sendo também influenciado pelo preço do arroz praticado no Estado do Mato Grosso.

Após a revisão da literatura acerca da transmissão de preços e de volatilidade, nota-se que é comum a aparição dos testes estatísticos: de raiz unitária de Dickey e Fuller (1981), de cointegração de Johansen (1988), de causalidade de Granger (1969), o VEC e testes de exogeneidade. Outro fato que se destaca é que, ao se analisarem diferentes séries temporais de preços de commodities, a maioria dos resultados apontou a ocorrência de transmissão de preços e relação de longo prazo. Observa-se também certa concordância dos pesquisadores quanto ao que se refere à transmissão de preço dos grandes mercados internacionais para os mercados domésticos.

Em relação às metodologias abordadas, a maioria dos pesquisadores optou pelo teste de raiz unitária com o intuito de determinar a ordem de integração das variáveis de interesse, bem como para verificar se as variáveis (séries temporais) são ou não estacionárias, enquanto que, para verificar o relacionamento entre as variáveis, os pesquisadores, geralmente, optam pelo teste de causalidade de Granger (1969) e cointegração de Johansen (1988). O uso do teste de cointegração justifica-se pela sua função de identificar a finalidade do possível relacionamento de longo prazo entre as variáveis. Além disso, o modelo VEC é bastante utilizado para a realização da análise econômica do relacionamento dos preços das variáveis, tanto de curto prazo, quanto de longo prazo.

\section{DADOS E PROCEDIMENTOS METODOLÓGICOS}

Em relação à abordagem de pesquisa, este trabalho caracteriza-se como quantitativa, constituindo-se da análise estatística de dados numéricos. Quanto à sua finalidade, é uma pesquisa do tipo descritiva, em que se apresentam características de determinado fenômeno que, no caso do presente estudo, trata-se da transmissão de preço internacional do trigo para os preços domésticos no Brasil. No contexto da aplicabilidade da pesquisa, 
espera-se que os resultados sejam utilizados como base para a tomada de decisão dos agentes da cadeia produtiva tritícola.

Dentre os meios de investigação desta pesquisa, iniciou-se com uma revisão de literatura (pesquisa bibliográfica) com ênfase em artigos publicados em periódicos qualificados e reconhecidos pelo alcance das bases científicas em que se encontram - incluindo qualificação no sistema de avaliação de periódicos da Coordenação de Aperfeiçoamento de Pessoal de Nível Superior (CAPES). Assim sendo, buscou-se dar robustez à fundamentação teórica acerca da temática abordada neste trabalho.

Quanto à caracterização dos dados, destaca-se que, nesta pesquisa, foram utilizados dados secundários obtidos em importantes fontes institucionais, dentre elas: (1) Centro de Estudos Avançados em Economia Aplicada (CEPEA) da Escola Superior de Agricultura Luiz de Queiroz (ESALQ) da Universidade do Estado de São Paulo (USP), que é uma fonte-base dos preços domésticos no Brasil - preços do trigo (preço médio entre os preços do Rio Grande do Sul e do Paraná; (2) IPEADATA - fonte-base do preços domésticos da farinha de trigo (média mensal do preço no Paraná); (3) Instituto de Economia Agrícola (IEA) fonte-base dos preços domésticos do pão francês (preço médio Região Metropolitana de São Paulo); (4) International Monetary Fund (IMF), fonte-base dos preços do trigo nos EUA (cotação mensal do Kansas); e (5) Bolsa de Cereales, que é a fonte-base dos preços do trigo na Argentina (preço médio entre as regiões de Buenos Aires, Quequén, Rosário, Bahía Blanca e Córdoba).

Quanto à dimensão temporal dos dados, o estudo compreendeu cotações de preços diários com início em fevereiro de 2004 e final em fevereiro de 2017. Com a finalidade de dar maior robustez, a análise da relação entre os preços foi transformada em séries mensais (média de preços mensais), visto que é comum que cotações em uma praça, em determinado dia, não sejam cotadas em outra praça, principalmente, quando há ocorrência de feriados, o que poderia gerar missing values em um significativo número de observações diárias. Uma vez transformados os dados em mensais, totalizando 785 observações (157 meses x 5 variáveis).

Para testar a ocorrência de transmissão de preços entre os mercados, optou-se por utilizar testes estatísticos complementares, iniciando-se com a verificação da estatística descritiva dos dados (medidas de tendência central, 
dispersão e correlação), seguida da avaliação da ordem de integração entre as séries. Com vistas ao alcance do objetivo principal da pesquisa, foi realizada a verificação da cointegração entre os preços-mercados. Os testes e análises estatísticos foram realizados com suporte-uso do software Eviews 8.0. No tratamento, transformação e seleção dos dados, foi utilizado o software Microsoft Excel.

Quanto ao tratamento estatístico para verificação da estacionariedade da série e avaliação da ordem de integração das mesmas, foi utilizado o teste de raiz unitária de Dickey-Fuller Aumentado (ADF) (1979, 1981). O teste é representado pelo sistema de equações (1), (2), (3), (4), de ADF, a fim de verificar a estacionariedade das séries de preços referente à cultura tritícola, representada pelas fórmulas:

$$
\begin{aligned}
& \Delta Y_{t}=\delta Y_{t-1}+u_{t} \\
& \Delta Y_{t}=\beta_{1}+\delta Y_{t-1}+u_{t} \\
& \Delta Y_{t}=\beta_{1}+\beta_{2} t+\delta Y_{t-1}+u_{t} \\
& \Delta Y_{t}=\beta_{1}+\beta_{2} t+\delta Y_{t-1}+\alpha_{i} \sum_{i=1}^{\rho} \Delta Y_{t-i}+u_{t}
\end{aligned}
$$

Em seguida, o teste de cointegração elaborado por Johansen (1988) foi utilizado para identificar o possível relacionamento de longo prazo entre as variáveis. Pretendeu-se, com esse teste, verificar a existência ou não de um relacionamento estocástico comum no longo prazo entre os preços domésticos e os preços internacionais do trigo. A equação de Johansen (1988) é modelada na seguinte equação:

$$
\Delta y_{t}=\mu+\Pi y_{t-1}+\ldots+\sum_{i=1}^{p-1} \Gamma y_{t-1}+\varepsilon_{t}
$$

Em que,

$$
\Pi=\sum_{i=1}^{p} A_{i}-1 \quad e \quad \Gamma=-\sum_{j=i+1}^{p} A_{j}
$$

Nesse caso, o coeficiente da matriz $\Pi$ posiciona-se como $r<n$, com $r \times n$ matrizes ( $\alpha$ e $\beta$ ), cada uma com posto $r$, de maneira que $\Pi=\alpha \beta$ e $\Pi=\beta^{\prime} y_{t}$ são estacionárias. Dessa maneira, tem-se: $r$ é o número de relações de cointegração; $\alpha$ é conhecido como parâmetro de ajustamento no vetor de correção do erro; e $\beta$ é um vetor de correção do erro. Para determinado $r$, a 
estimativa da máxima verossimilhança da matriz $\beta$ determina a combinação de $y_{t-1}$ que resulta $r$ correlações canônicas maiores entre $\Delta y_{t}$ e $y_{t-1}$, depois das correções de diferenças de defasagem e variáveis determinísticas, caso constatadas. A significância da razão de verossimilhança das correlações canônicas é estimada pelo teste traço, como segue.

$$
\lambda_{\text {traço }}=-T \sum_{i=r+1}^{n} \ln \left(1-\hat{\lambda}_{t}\right)
$$

Em que: $T$ é a dimensão da amostra e $\hat{\lambda}_{r+1}$ é a $i$-ésima maior correlação canônica. O teste traço afere a hipótese nula de que o número de vetores de cointegração é menor ou igual a $r$ versus a hipótese alternativa de que o número de vetores de cointegração é maior que $r$. As hipóteses do teste são: $r=0$, não há cointegração; $\mathrm{e}, r \geq 1$, há cointegração. O nível de significância estatística estabelecido para rejeição ou não da hipótese foi de 5\%, com valores críticos definidos, conforme Johansen e Juselius (1990).

Uma vez verificada a cointegração entre as séries, aplicou-se, na sequência, o modelo vetorial autorregressivo de correção de erro (VEC), o que possibilitou a realização da análise de existência de relacionamento econômico de longo prazo entre os preços internacionais e os preços domésticos. 0 modelo VEC é representado pelo sistema equacional a seguir:

$$
\begin{aligned}
& \Delta S_{t}=c_{s}+\sum_{i=1}^{k} \beta_{s i} \Delta S_{t-i}+\sum_{i=1}^{k} \beta_{s i} \Delta F_{t-i}+\gamma_{s} Z_{t-1}+\mu_{s t} \\
& \Delta F_{t}=c_{f}+\sum_{i=1}^{k} \beta_{f i} \Delta S_{t-i}+\sum_{i=1}^{k} \beta_{f i} \Delta F_{t-i}-\gamma_{f} Z_{t-1}+\mu_{f t}
\end{aligned}
$$

Em que: $c$ é o intercepto; $\beta_{s i}$ e $\beta_{f i}$ são parâmetros positivos; $\mu_{s t}$ e $\mu_{f t}$ são vetores, aleatoriamente distribuídos de forma idêntica e independente; $\gamma_{s}$ e $\gamma_{f}$ são parâmetros positivos; e $Z_{t-1}$ é o termo de correção do erro que afere como a variável dependente preço doméstico se ajusta aos desvios dos períodos anteriores promovidos pelo "equilíbrio" no longo prazo com o preço internacional, como especificado na equação 9:

$S_{t-1}=\alpha+\beta F_{t}+Z_{t-1}$ 
Nessa equação, $\alpha$ refere-se ao elemento de ajustamento do vetor de cointegração; e $\beta$ é o vetor de cointegração. Assim, $\alpha$ e $\beta$ correspondem ao modelo vetorial autorregressivo na primeira diferença do sistema de equações 8 , enquanto $\gamma_{s}$ e $\gamma_{f}$ se referem à velocidade de ajustamento. Dessa forma, quanto maior $\gamma_{s}$, maior será a velocidade de ajustamento de $S_{t}$ aos desvios anteriores, o que se deve ao equilíbrio do relacionamento no longo prazo.

\section{ANÁLISE DE RESULTADOS E DISCUSSÕES}

Na Figura 1, encontram-se as séries de preços médios mensais do trigo no Brasil, na Argentina e nos Estados Unidos da América (EUA), cotados em dólares por tonelada (US\$/ton). Nota-se, figura 1 , que os preços do trigo brasileiro e norte-americano apresentam comportamento muito similar e, salvo curtos espaços de tempo, geralmente, encontram-se afastados dos preços do trigo argentino.

Ao observar ainda a Figura 1, chama a atenção o período 2010-2014, quando os preços do trigo argentino apresentaram maior volatilidade, ora se posicionando muito abaixo dos preços do trigo norte-americano, ora se encontrando acima, como é o caso do ano de 2013, em que, na maior parte do tempo, foi superior ao preço do trigo nos EUA. É possível inferir que a política intervencionista no mercado agrícola, a volatilidade do câmbio e o cenário de incertezas da economia argentina sejam alguns dos fatores responsáveis pela volatilidade do preço do trigo argentino no período 20102014. Outro comportamento importante a ser considerado diz respeito à aproximação dos preços do trigo argentino aos preços do trigo norteamericano a partir do ano de 2015, o que pode ser reflexo das alterações nas políticas adotadas pelo governo argentino quanto aos mercados agrícolas. 


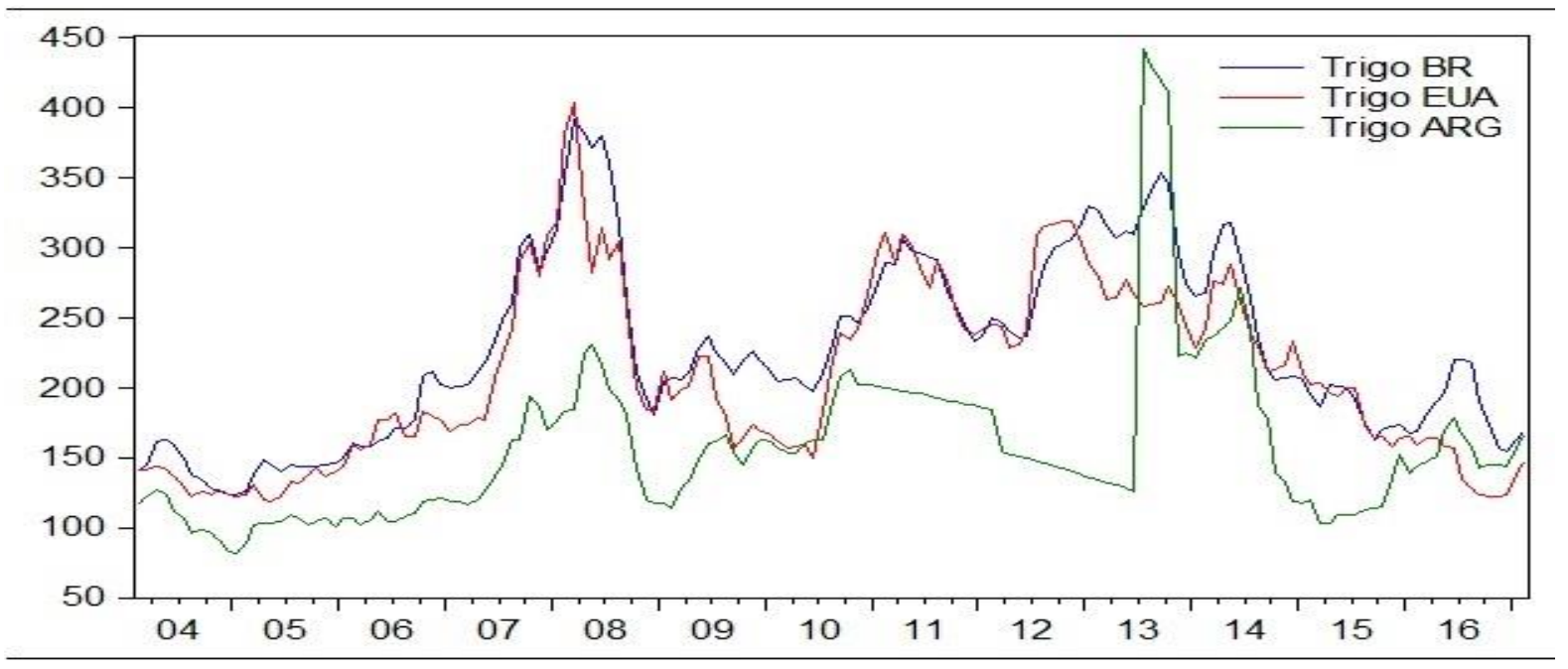

Gráfico 1: Preços do trigo brasileiro, argentino e norte-americano no período fev.2004/fev.2017.

Fonte: Dados da pesquisa.

Conforme os dados da Tabela 1, disposta abaixo, a qual se compõe das estatísticas descritivas dos preços em US\$/ton, do trigo, da farinha de trigo e do pão francês nos mercados em estudo, é possível visualizar as medidas de tendência central, dispersão e distribuição. Nessa tabela, é possível observar que a média de preços do trigo brasileiro é bastante superior à média de preços do trigo argentino e norte-americano (o que já era esperado, uma vez que o Brasil é o maior importador dessa commodity na América Latina). Mas, ao verificar a dispersão dos preços do trigo, tem-se um desvio padrão de $28 \%$, $38 \%$ e $31 \%$ sobre a média de preço, o que demonstra a maior volatilidade dos preços do trigo argentino quando comparada à volatilidade dos preços do trigo norte-americano e brasileiro.

O desvio padrão da farinha de trigo e do pão francês também é de $31 \%$, ou seja, apresenta comportamento coerente com os valores observados para o trigo norte-americano e brasileiro. Assim, ao analisar a distribuição dos dados pelo teste de Jarque-Bera, rejeita-se a hipótese nula de normalidade. A não normalidade das distribuições de dados também é evidenciada pelas estatísticas de assimetria e curtose.

Tabela 1: Estatística descritiva dos preços do trigo, farinha de trigo e pão francês no Brasil e os preços do trigo na Argentina e EUA

\begin{tabular}{lccccc}
\hline \multirow{2}{*}{ Média } & Trigo BR & Trigo ARG & Trigo EUA & Farinha BR & Pão BR \\
\cline { 2 - 5 } & 226,6415 & 156,5718 & 209,0508 & 535,5570 & 2937,1060
\end{tabular}




\begin{tabular}{lccccc} 
Mediana & 211,3060 & 145,0561 & 199,1966 & 510,9677 & 3161,1110 \\
Máximo & 391,0943 & 441,1053 & 403,8129 & 1012,4480 & 4416,6670 \\
Mínimo & 123,6415 & 81,6740 & 119,1545 & 259,0985 & 1221,8650 \\
DP & 64,7786 & 59,0365 & 63,9014 & 166,0057 & 912,1725 \\
Assimetria & 0,4647 & 2,4584 & 0,4686 & 0,5065 & $-0,3790$ \\
Curtose & 2,3649 & 11,6652 & 2,3540 & 2,6730 & 1,7759 \\
Jarque-Bera & 8,2902 & 649,3260 & 8,4748 & 7,4121 & 13,5611 \\
$p$-valor & 0,0158 & 0,0000 & 0,0144 & 0,0246 & 0,0011 \\
\hline
\end{tabular}

Notas: (DP) Desvio Padrão, (BR) Brasil, (ARG) Argentina, (EUA) Estados Unidos da América.

Fonte: Dados da pesquisa.

A correlação linear entre os preços brasileiros do trigo, farinha de trigo e pão francês, bem como entre os preços do trigo argentino e norte-americano, é apresentada na Tabela 2. A partir dos dados da tabela, afere-se, com base nos resultados, que a correlação entre os preços do trigo brasileiro é forte e positiva com os preços do trigo norte-americano $(0,92)$ e moderada e positiva com os preços do trigo argentino $(0,68)$.

Essa correlação é corroborada quando se observam as associações positivas dos preços da farinha de trigo e do pão francês com os preços do trigo argentino que são, respectivamente, 0,72 e 0,56, e com os preços do trigo norte-americano, que são de 0,79 e 0,59. Nesse caso, constatou-se também a existência de uma relação linear mais forte entre os preços brasileiros e norte-americanos quando comparados com a associação com os preços argentinos. A correlação positiva significativa entre os preços do trigo e de seus principais derivados no mercado brasileiro com os preços do trigo norte-americano e argentino implica na possibilidade de transmissão dos preços do mercado externo para o mercado interno.

Tabela 2: Correlação linear entre os preços brasileiros do trigo, farinha de trigo e pão francês, e preços do trigo da Argentina e EUA

\begin{tabular}{cccccc}
\hline Série de Preços & Trigo BR & Trigo ARG & Trigo EUA & Farinha BR & Pão BR \\
\hline Trigo BR & 1,0000 & $0,6786^{*}$ & $0,9213^{*}$ & $0,9181^{*}$ & $0,6440^{*}$ \\
Trigo ARG & & 1,0000 & $0,5168^{*}$ & $0,7108^{*}$ & $0,5688^{*}$ \\
Trigo EUA & & & 1,0000 & $0,7927^{*}$ & $0,5911^{*}$ \\
Farinha BR & & & & 1,0000 & $0,6777^{*}$ \\
Pão BR & & & & & 1,0000 \\
\hline
\end{tabular}

Notas: (BR) Brasil, (ARG) Argentina, (EUA) Estados Unidos da América e $\left(^{*}\right.$ ) significante estatisticamente ao nível de $1 \%$.

Fonte: Dados da pesquisa. 
Como primeiro passo para verificação da hipótese de transmissão de preço entre os mercados externo e doméstico, aplicou-se o teste ADF, a fim de apurar a presença ou não de raiz unitária, bem como para verificar a ordem de integração entre as séries de preços, resultados esses expostos na Tabela 3.

De acordo com o teste de raiz unitária ADF, tanto em nível, quanto com intercepto, e com tendência linear, o valor da estatística $t$ apresentou resultados maiores que o valor crítico ao nível de 1\%, 5\% e 10\% (ver equações 1, 2 e 3), ou seja, não se rejeitou a hipótese nula de presença de raiz unitária, o que indica que as séries são não estacionárias em nível. Em seguida, verificou-se que essas séries não possuem raiz unitária na primeira diferença, ou seja, quando transformada em retornos, as séries são estacionárias e integradas de primeira ordem. Ao avaliar a estatística d de Durbin e Watson, cujos valores se aproximam muito de 2 , notou-se que as séries temporais de preços não apresentam problemas de autocorrelação na $1^{a}$ diferença.

Tabela 3: Teste de raiz unitária de Dickey-Fuller aplicado nos preços do trigo brasileiro, argentino e norte-americano, e nos preços da farinha de trigo e pão francês do Brasil

\begin{tabular}{|c|c|c|c|c|c|c|c|c|}
\hline \multirow{2}{*}{$\begin{array}{c}\text { Série de } \\
\text { Preços }\end{array}$} & \multirow{2}{*}{$\begin{array}{c}\text { Equação } \\
\text { ADF }\end{array}$} & \multirow{2}{*}{$\begin{array}{c}\text { Estatística } \\
\text { ADF }\end{array}$} & \multicolumn{3}{|c|}{ Valores Críticos } & \multirow{2}{*}{ AIC } & \multirow{2}{*}{ SC } & \multirow{2}{*}{ DW } \\
\hline & & & $1 \%$ & $5 \%$ & $10 \%$ & & & \\
\hline \multirow{4}{*}{ Trigo BR } & $\mathrm{N}$ & $-0,6112^{\mathrm{ns}}$ & $-2,5801$ & $\begin{array}{c}- \\
1,9429\end{array}$ & $\begin{array}{c}- \\
1,6153\end{array}$ & 7,9041 & 7,9633 & 2,0143 \\
\hline & I & $-2,8531 \mathrm{~ns}$ & $-3,4728$ & 2,8801 & $\begin{array}{c}- \\
2,5767\end{array}$ & 7,8931 & 7,9520 & 1,8252 \\
\hline & IT & $-2,7738^{\mathrm{ns}}$ & $-4,0183$ & $\begin{array}{c}- \\
3,4391\end{array}$ & $\begin{array}{c}- \\
3,1439\end{array}$ & 7,9058 & 7,9843 & 1,8272 \\
\hline & $1^{\mathrm{a} D \text { Dif }}$ & $-7,1135^{*}$ & $-2,5801$ & $1, \overline{-}$ & $\begin{array}{c}- \\
1,6153\end{array}$ & 7,8936 & 7,9331 & 2,0152 \\
\hline \multirow{4}{*}{ Trigo ARG } & $\mathrm{N}$ & $-0,6410^{n s}$ & $-2,5803$ & 1,9429 & $\begin{array}{c}- \\
1,6153\end{array}$ & 9,6531 & 9,7525 & 1,9942 \\
\hline & I & $-2,7136^{\mathrm{ns}}$ & $-3,4737$ & 2,8805 & $\begin{array}{c}- \\
2,5769\end{array}$ & 9,6196 & 9,739 & 1,9675 \\
\hline & IT & $-2,8652$ ns & $-4,0196$ & $\begin{array}{c}- \\
3,4397\end{array}$ & $\begin{array}{c}- \\
3,1442\end{array}$ & 9,6264 & 9,7657 & 1,9615 \\
\hline & $1^{\mathrm{a} D i f}$ & $-9,2171^{*}$ & $-2,5803$ & 1,9429 & $\begin{array}{c}- \\
1,6153\end{array}$ & 9,6427 & 9,7223 & 1,9976 \\
\hline
\end{tabular}


Continuação

\begin{tabular}{|c|c|c|c|c|c|c|c|c|}
\hline \multirow{4}{*}{ Trigo EUA } & $\mathrm{N}$ & $-0,6160 \mathrm{~ns}$ & $\begin{array}{c}- \\
2,5800\end{array}$ & 1,9429 & $\begin{array}{c}- \\
1,6153\end{array}$ & 8,6259 & 8,6651 & 1,9491 \\
\hline & I & $-2,2791 \mathrm{~ns}$ & $\begin{array}{c}- \\
3,4728\end{array}$ & 2,8801 & 2,5767 & 8,6076 & 8,6665 & 1,9584 \\
\hline & IT & $-2,1413^{n s}$ & $\begin{array}{c}- \\
4,0183\end{array}$ & $\begin{array}{c}- \\
3,4391\end{array}$ & 3,1439 & 8,6201 & 8,6986 & 1,9576 \\
\hline & $1^{\mathrm{a} D \text { Dif }}$ & $-10,0295^{*}$ & $\begin{array}{c}- \\
2,5800 \\
\end{array}$ & $\begin{array}{c}- \\
1,9429\end{array}$ & $\begin{array}{c}- \\
1,6153\end{array}$ & 8,6154 & 8,6351 & 1,9489 \\
\hline \multirow{4}{*}{ Farinha BR } & $\mathrm{N}$ & $-0,5478^{n s}$ & $\begin{array}{c}- \\
2,5800\end{array}$ & $\begin{array}{c}- \\
1,9429\end{array}$ & $\begin{array}{c}- \\
1,6153\end{array}$ & 10,4695 & 10,5088 & 2,0230 \\
\hline & I & $-2,5270 \mathrm{~ns}$ & $\begin{array}{c}- \\
3,4728\end{array}$ & 2,8801 & $\begin{array}{c}- \\
2,5767\end{array}$ & 10,4430 & 10,5019 & 2,0433 \\
\hline & IT & $-2,4448 \mathrm{~ns}$ & $\begin{array}{c}- \\
4,0183\end{array}$ & $\begin{array}{c}- \\
3,4391\end{array}$ & $\begin{array}{c}- \\
3,1439\end{array}$ & 10,4556 & 10,5341 & 2,0451 \\
\hline & $1^{\mathrm{a} D \text { Dif }}$ & $-9,3994^{*}$ & $\begin{array}{c}- \\
2,5800\end{array}$ & $\begin{array}{c}- \\
1,9429\end{array}$ & $\stackrel{-}{-6153}$ & 10,4586 & 10,4782 & 2,0209 \\
\hline \multirow{4}{*}{ Pão BR } & $\mathrm{N}$ & $0,5245^{\text {ns }}$ & $\begin{array}{c}- \\
2,5800\end{array}$ & 1,9429 & $\begin{array}{c}- \\
1,6153\end{array}$ & 13,5467 & 13,5860 & 1,9947 \\
\hline & I & $-1,7676^{\mathrm{ns}}$ & $\begin{array}{c}- \\
3,4728\end{array}$ & 2,8801 & $\begin{array}{c}- \\
2,5767\end{array}$ & 13,5333 & 13,5923 & 1,9924 \\
\hline & IT & $-2,3171^{\mathrm{ns}}$ & 4, & $\begin{array}{c}- \\
3,4389\end{array}$ & 3,1438 & 13,5511 & 13,6098 & 2,3233 \\
\hline & $1^{a}$ Dif & $-15,0818^{*}$ & 2,5800 & $1, \overline{-}$ & $\begin{array}{c}- \\
1,6153\end{array}$ & 13,5356 & 13,5552 & 1,9930 \\
\hline
\end{tabular}

Notas: (BR) Brasil, (ARG) Argentina, (EUA) Estados Unidos da América, (N) equação do teste em nível, (I) equação do teste com intercepto, (IT) equação do teste com intercepto e tendência linear, ( $1^{a}$ Dif) equação do teste na primeira diferença, (AIC) critério de informação de Akaike, (SBC) critério de informação de Schwarz, (DW) estatística $d$ de Durbin e Watson, $\left({ }^{*}\right)$ Estatisticamente significante ao nível de $1 \%$, (ns) Estatisticamente não significativo.

Fonte: Dados da pesquisa.

Após a realização do teste de raiz unitária e verificação da ordem de integração entre as séries de preços, definiu-se o número de defasagens ótimas para o teste de cointegração de Johansen (1988), com a finalidade de verificar o equilíbrio da relação de longo prazo entre os preços brasileiros do trigo e derivados e os preços do trigo no mercado argentino e norte-americano.

$\mathrm{Na}$ Tabela 4, encontram-se os valores dos critérios de informação de Akaike, Schwarz e Hannan-Quinn utilizados como base de definição do número de defasagens ótimas para o teste de cointegração. Frente aos resultados obtidos, os critérios sugerem um número ótimo de duas defasagens para as séries, exceto, da relação entre os preços do trigo brasileiro e norte-americano, cuja defasagem ótima é cinco. 
Tabela 4: Defasagens ótimas entre os preços brasileiros do trigo, farinha de trigo e pão francês e preços internacionais do trigo

\begin{tabular}{cccccc}
\hline Preços & Cointegração & Defasagens & AIC & SC & HQ \\
\hline \multirow{2}{*}{ Trigo BR } & Trigo ARG & 2 & $-3,2504$ & $-3,1290$ & $-3,2011$ \\
& Trigo EUA & 5 & $-5,9783$ & $-5,8470$ & $-5,9190$ \\
\cline { 2 - 6 } Farinha BR & Trigo ARG & 2 & $-2,3324$ & $-2,2920$ & $-2,3160$ \\
& Trigo EUA & 2 & $-4,4815$ & $-4,3897$ & $-4,4321$ \\
\cline { 2 - 6 } Pão BR & Trigo ARG & 2 & $-2,5521$ & $-2,5116$ & $-2,5356$ \\
& Trigo EUA & 2 & $-4,7405$ & $-4,6190$ & $-4,6912$ \\
\hline
\end{tabular}

Notas: (BR) Brasil, (ARG) Argentina, (EUA) Estados Unidos da América, (AIC) critério de informação de Akaike, (SBC) critério de informação de Schwarz, (HQ) critério de informação de Hannan-Quinn.

Fonte: Dados da pesquisa

Definido o número ótimo de defasagens, procedeu-se à realização do teste de cointegração, como mostra a Tabela 5, tendo sido definido em $5 \%$ de significância estatística como base para rejeição ou não da hipótese de cointegração. Assim sendo, ao observar os valores críticos e os valores do teste traço, foi rejeitada a hipótese nula da existência de nenhum vetor de cointegração entre as séries de preços domésticos e preços internacionais. É importante ressaltar que a condição para que haja uma tendência estocástica comum entre as séries de preços é a existência do vetor de cointegração (portanto, a hipótese de vetor nulo foi rejeitada, ou seja, há pelo menos um vetor de cointegração). Diante disso, os resultados apresentados na Tabela 4 sugerem que a série de preços possui relação comum-equilibrada de longo prazo.

A constatação de cointegração entre os preços de commodities domésticas e seus respectivos preços internacionais também pode ser percebida no estudo de W. Silva et al. (2003); Corso et al. (2006); Tonin e Barczsz (2007) e Freitas et al. (2012). 


\section{Tabela 5: Teste traço para verificação de cointegração entre os preços do trigo, farinha de trigo, pão francês em relação aos preços da Argentina e EUA}

\begin{tabular}{|c|c|c|c|c|c|c|}
\hline \multirow{2}{*}{$\begin{array}{l}\text { Variável de } \\
\text { Cointegração }\end{array}$} & \multirow{2}{*}{$\begin{array}{l}\text { Série de } \\
\text { Preços }\end{array}$} & \multirow{2}{*}{$\begin{array}{l}\text { Vetores de } \\
\text { Cointegração }\end{array}$} & \multirow[b]{2}{*}{ Eigenvalue } & \multicolumn{3}{|c|}{ Teste Traço } \\
\hline & & & & Traço & $\begin{array}{c}\text { Valor Crítico } \\
(5 \%)\end{array}$ & p-valor \\
\hline \multirow{4}{*}{ Trigo BR } & \multirow{2}{*}{ Trigo ARG } & 0 & 0,2615 & 86,9747 & 15,4947 & 0,0000 \\
\hline & & 1 & 0,2331 & 40,6028 & 3,8415 & 0,0000 \\
\hline & \multirow{2}{*}{ Trigo EUA } & 0 & 0,2007 & 52,2756 & 15,4947 & 0,0000 \\
\hline & & 1 & 0,1170 & 18,6718 & 3,8415 & 0,0000 \\
\hline \multirow{4}{*}{ Farinha BR } & \multirow{2}{*}{ Trigo ARG } & 0 & 0,2915 & 90,6296 & 15,4947 & 0,0000 \\
\hline & & 1 & 0,2194 & 37,8947 & 3,8415 & 0,0000 \\
\hline & \multirow{2}{*}{ Trigo EUA } & 0 & 0,3012 & 90,1542 & 15,4947 & 0,0000 \\
\hline & & 1 & 0,2061 & 35,3153 & 3,8415 & 0,0000 \\
\hline \multirow{4}{*}{$\begin{array}{c}\text { Pão francês } \\
\text { BR }\end{array}$} & \multirow{2}{*}{ Trigo-ARG } & 0 & 0,2939 & 94,7223 & 15,4947 & 0,0000 \\
\hline & & 1 & 0,2375 & 41,4799 & 3,8415 & 0,0000 \\
\hline & \multirow{2}{*}{ Trigo-EUA } & 0 & 0,2891 & 93,5925 & 15,4947 & 0,0000 \\
\hline & & 1 & 0,2370 & 41,3862 & 3,8415 & 0,0000 \\
\hline
\end{tabular}

Notas: (BR) Brasil, (ARG) Argentina, (EUA) Estados Unidos da América.

Fontes: Dados da pesquisa.

Após a verificação do relacionamento estocástico comum entre as séries de preços no longo prazo e a definição a respeito da hipótese de existência de, no mínimo, um vetor de cointegração, o estudo segue com a aplicação do modelo VEC. Na Tabela 6, encontram-se as equações de cointegração entre as séries e os respectivos modelos vetoriais autorregressivos com a inclusão do mecanismo de correção de erro.

De acordo com os dados da Tabela 6, observa-se que os preços convergem no curto prazo. Partindo-se do pressuposto de que $\beta$ representa a elasticidade de ajustamento entre os preços no curto prazo e $a$ representa a velocidade de ajuste dos preços, ou seja, mostra a velocidade de ajustamento dos preços em direção ao equilíbrio no longo prazo.

Diante dos valores dos coeficientes $\beta$, nota-se que, no curto prazo, a alteração de $1 \%$, na variação do preço do trigo argentino transfere alterações de $1,34 \%, 0,59 \%$ e $0,23 \%$ para a variação dos preços domésticos-brasileiros do trigo, farinha de trigo e pão francês, respectivamente. Já na hipótese de alterações de $1 \%$ na variação do preço trigo norte-americano no curto prazo, essas são transferidas em conjunto para variações na ordem de $0,70 \%, 1,29 \%$ e 0,54\% para variações dos preços domésticos-brasileiros do trigo, farinha de trigo e pão francês. Tais constatações sugerem, portanto, que as séries se relacionam de forma inelástica. 
Os resultados da Tabela 6 indicam que o trigo brasileiro é mais influenciado pelo trigo argentino, corroborando o fato do Brasil ser o maior importador do commodity argentina, tanto do grão para o consumo, como da semente voltada ao plantio, conforme dados da ABITRIGO (2016). Já em relação à farinha de trigo, notou-se que o grão norte-americano é o que apresenta maior influência sobre os derivados do trigo brasileiro, mais precisamente, na farinha de trigo e no pão francês, o que corrobora o fato de o trigo proveniente dos EUA ter como finalidade principal o processamento e consumo, influenciando, portanto, mais os derivados do que propriamente o trigo brasileiro.

Os resultados da pesquisa mostraram que o preço do pão é o que sofre menor influência dos preços do trigo argentino e norte-americano, principalmente, se comparado aos preços brasileiros do trigo e da farinha de trigo. Vale ressaltar que os preços do pão francês no mercado brasileiro sofrem mais influências das alterações ocorridas nos preços do trigo norte-americano do que das alterações dos preços do trigo argentino. Esse resultado corrobora a influência dos preços do trigo norte-americano sobre a farinha de trigo brasileira, quando comparada com o impacto gerado, devido às alterações no preço do trigo argentino. Sobre esses resultados pesa o fato de a farinha de trigo ser a principal matéria-prima para fabricação do pão francês.

Tabela 6: Equação de cointegração e modelos vetoriais autorregressivos com a inclusão do mecanismo de correção de erro

\begin{tabular}{ccccccc}
\hline & Trigo & Trigo & Farinha & Farinha & Pão & Pão BR/EUA \\
BR/ARG & BR/EUA & BR/ARG & BR/EUA & BR/ARG & \\
\hline A & 0,0124 & $-1,0200$ & $-0,4130$ & $-0,5519$ & $-0,8686$ & $-1,0099$ \\
B & $-1,3419$ & $-0,7000$ & $-0,5938$ & $-1,2851$ & $-0,2314$ & $-0,5388$ \\
Erro Padrão & $-0,1740$ & $-0,0900$ & $-0,0797$ & $-0,1665$ & $-0,0452$ & $-0,0998$ \\
Estatística $t$ & {$[-7,7105]$} & {$[-7,4433]$} & {$[-7,4543]$} & {$[-7,7195]$} & {$[-5,1156]$} & {$[-5,3988]$} \\
\hline Constante & 0,0001 & 0,0000 & $-0,0001$ & 0,0001 & 0,0008 & 0,0009 \\
& {$[0,0223]$} & {$[0,3773]$} & {$[-0,0137]$} & {$[0,0158]$} & {$[0,1379]$} & {$[0,1510]$} \\
Equação & 0,0124 & $-1,0200$ & $-0,4130$ & $-0,5519$ & $-0,8686$ & $-1,0099$ \\
& {$[0,4136]$} & {$[-4,8950]$} & {$[-4,4649]$} & {$[-5,1622]$} & {$[-6,4230]$} & {$[-6,4824]$} \\
D(BR(-1)) & $-0,1460$ & 0,5100 & $-0,3426$ & $-0,2809$ & $-0,2609$ & $-0,1696$ \\
& {$[-1,7681]$} & {$[2,9316]$} & {$[-3,7827]$} & {$[-2,9863]$} & {$[-2,3183]$} & {$[-1,3651]$} \\
D(BR(-2)) & $-0,2028$ & 0,4100 & $-0,1970$ & $-0,1622$ & $-0,1230$ & $-0,1077$ \\
& {$[-2,4997]$} & {$[2,5561]$} & {$[-2,5491]$} & {$[-2,1030]$} & {$[-1,5757]$} & {$[-1,3456]$} \\
D(BR(-3)) & & 0,3300 & & & & \\
& & {$[2,2552]$} & & & &
\end{tabular}




\begin{tabular}{|c|c|c|c|c|c|c|}
\hline $\mathrm{D}(\mathrm{BR}(-5))$ & & $\begin{array}{c}{[0,5322]} \\
-0,0200 \\
{[-0,1902]}\end{array}$ & & & & Continuação \\
\hline$R^{2}$ & 0,0583 & 0,3500 & 0,3663 & 0,4037 & 0,5847 & 0,5982 \\
\hline$R^{2 A j u s t a d o}$ & 0,0262 & 0,3000 & 0,3448 & 0,3834 & 0,5706 & 0,5845 \\
\hline AIC & $-2,7970$ & $-3,0700$ & $-1,9774$ & $-2,0382$ & $-2,3266$ & $-2,3595$ \\
\hline SBC & $-2,6782$ & $-2,8300$ & $-1,8586$ & $-1,9194$ & $-2,2078$ & $-2,2407$ \\
\hline
\end{tabular}

Notas: (BR) Brasil, (ARG) Argentina, (EUA) Estados Unidos da América. (LV) Logverossimilhança, (AIC) critério de informação de Akaike, (SBC) critério de informação de Schwarz e [.] estatística t.

Fonte: Dados da pesquisa.

Os parâmetros de a mostram que a velocidade de ajustamento dos preços apresentou um desequilíbrio de curto prazo no preço do trigo da argentina, sendo a velocidade do ajuste lenta no preço do trigo brasileiro, uma vez que o ajuste é de apenas $-1,24 \%$, ou seja, o reajuste exige um extenso período e lentamente irá se ajustar em longo prazo. Ao considerar a velocidade de ajuste para a farinha de trigo $(41,30 \%)$ e para o pão francês $(86,86 \%)$, a velocidade de ajustamento no longo prazo é mais rápida.

A baixa velocidade de ajustamento no longo prazo, quando há um desequilíbrio de curto prazo no preço argentino, refere-se ao fato de que, se houver restrições de oferta da Argentina, o Brasil pode buscar trigo de outros países, como, por exemplo, no Uruguai e Paraguai, no caso de sementes, conforme dados da ABITRIGO (2016), além de contar com diversas opções, como de países que ofertam trigo em grão. Esse resultado corrobora o estudo de Margarido et al. (2007), que também encontrou velocidade baixa de ajustamento do preço interno no Brasil em relação ao preço argentino.

A redução do desequilíbrio de curto prazo dos preços do trigo norteamericano para o equilíbrio no longo prazo em relação aos preços do trigo brasileiro (102\%), farinha de trigo $(55,19 \%)$ e pão francês $(101 \%)$ se ajusta rapidamente.

Em suma, após o teste de transmissão e sua respectiva análise, foram verificadas evidências de um relacionamento estocástico comum no longo prazo entre os preços internos e externos no curto prazo. Verifica-se também, diante dos resultados encontrados, que o preço do trigo é mais sensível ao preço do trigo argentino. Em contrapartida, a farinha de trigo se mostra sensível frente ao preço do trigo norte-americano. Esses resultados reforçam a constatação do estudo de Mundlak e Larson (1992), os quais encontraram o 
resultado de transmissão de preço dos mercados externos para os mercados internos.

\section{CONCLUSÕES}

Dada a importância de informações para tomada de decisões mais precisas dos agentes da cadeia produtiva do trigo no Brasil, este estudo teve como objetivo analisar a volatilidade e a transmissão do preço do trigo internacional para os preços domésticos do trigo e derivados no Brasil, abrangendo uma série temporal entre fevereiro de 2004 a fevereiro de 2017.

Em relação à finalidade do estudo, destaca-se, primeiramente, a constatação da cointegração entre os preços do trigo e derivados domésticos (Brasil) e os preços internacionais (Argentina e EUA), ou seja, evidenciou-se o relacionamento estocástico comum entre as séries de preços no longo prazo. Nesse sentido, concluiu-se ainda que, no curto prazo, o preço do trigo brasileiro é, significativamente, impactado por variações no preço do trigo argentino, o que também se aplica à farinha de trigo diante das alterações nos preços do trigo norte-americano.

Apesar de os resultados apontarem para a possibilidade da existência de transmissão de preços dos mercados internacionais (argentino e norteamericano) para o mercado brasileiro, concluiu-se que o trigo brasileiro é mais influenciado pelos preços do trigo argentino, ao passo que os derivados do trigo brasileiro (farinha de trigo e pão francês) sofrem maior influência das variações ocorridas nos preços do trigo norte-americano, conforme evidenciado nos resultados dos testes de cointegração e aplicação do modelo VEC nas séries de preços.

Diante dos resultados da pesquisa, concluiu-se que um choque nos preços do trigo argentino no curto prazo é transferido para os preços do trigo brasileiro, porém tem-se um extenso período de tempo de ajuste rumo ao equilíbrio da relação entre os mesmos. Infere-se que isso ocorra pelo fato de o Brasil, geralmente, recorrer a outros mercados, principalmente, ao norteamericano, para importação da commodity.

Ademais, os resultados deste estudo mostraram a relação estatisticamente significativa entre os preços do trigo no mercado argentino e 
norte-americano e os preços do trigo e derivados no mercado brasileiro, o que reforça a importância da atenção dos agentes dessa cadeia produtiva quanto às informações advindas desses mercados, em especial, aquelas que estão diretamente ligadas a possíveis alterações nos preços de curto e longo prazo.

Ao fim desta pesquisa, sugere-se que futuros estudos sejam realizados com outras commodities agrícolas e produtos derivados, além de analisados outros mercados. Além disso, espera-se que outros modelos de análise sejam aplicados, o que permitiria uma comparação com os resultados desta e de outras pesquisas do gênero, contribuindo tanto para a perspectiva de avaliação dos resultados, como também para o avanço no campo científico e para a tomada de decisões na gestão de risco e comercialização de commodities agrícolas e seus derivados. 


\section{REFERÊNCIAS}

Associação Brasileira da Indústria do Trigo. 2016. Estatísticas: Confira os principais dados e informações sobre o mercado do trigo. Recuperado em 16 de março, 2016, de http:// http://www.abitrigo.com.br/estatisticas.php

Alves, F. L. B., Silva F. J. T., Nascimento, J. A. B. do., Mascarenhas, E. P. \& Djau, M. A. (2014, abril/junho). Análise de convergência entre os preços de mercado do trigo entre Estados Unidos da América, Argentina e Brasil no período de 2004 a 2012. Revista de Economia e Administração, 13 (2), 235250.

Alves, L. R. A., Cardoso, C. E. L., Felipe, F. I. \& Campion, M. T. (2015) Causalidade e transmissão entre os preços de mandioca, trigo, milho e seus derivados no paraná. Revista de economia e agronegócio, 4 (3), 313-342.

Barbosa, M. Z., Margarido, M. A. \& Nogueira, S. Jr. (2002, julho/dezembro). Análise da elasticidade de transmissão de preços no mercado brasileiro de algodão. Nova Economia, 12, 2, 79-108.

Bessler, D. A., Yang, J. \& Wongcharupan, M. (2003, february) Price dynamics in the international wheat market: modeling with error correction and directed acyclic graphs. Journal of Regional Science, 43, 1, 1-33.

Block, A., Coronel, D. A. \& Veloso, G. de O. (2012, maio/agosto). Análise da transmissão de preços no setor sucroalcooleiro brasileiro. Revista eletrônica de estratégia e negócios, 5, 2, 120-137. Recuperado em 29 de maio, 2016, de http://portaldeperiodicos.unisul.br/index.php/EeN/index

Brown, C. J. \& Curci, R. (2002). Mexican peso futures and exchange rate volatility. Latin American Business Review, 3, 1, 75-90 Disponível em: http://www.tandfonline.com/doi/pdf/10.1300/J140v03n01_04. Acesso em: 29/05/2016.

Coopération Internationale pour le Développementet la Solidarity. Volatilidade dos preços dos alimentos. 2011. Recuperado em 14 de março, 2016, de http://docplayer.com.br/47072-Volatilidade-dos-precos-dosalimentos.html

Coronel, D. A., Amorim, A. L., Sousa, E. P. de. \& Lima, J. E. de. (2010) Integração e transmissão de preços entre os mercados de trigo argentino e internacional. Pesquisa \& Debate, 21, 2 (38), 279-305.

Corso, J. M. D., Silva, W. V. da. \& Duclós, L. C. (2006, julho/setembro) Avaliação do processo de transmissão dos preços da soja praticados nos mercados físico brasileiro e norte-americano. Revista de Negócios, 11, 3, 61-72.

Dickey, D.A. \& Fuller, W. A. (1981, julho) Likelihood ratio statistics for autoregressive time series with a unit root. Econometrica, 49, 4, 10571072. 
Dickey, D.A \& Fuller, W. A. (1979). Distribution of the estimator for auto regressive time series with a unit root. Journal of the American Statistical Association, 74, 366, 427-431.

Freitas, C. O. de., Lírio, V. S. \& Coronel, D. A. (2012, janeiro/junho) Análise da transmissão de preço do fumo entre os estados produtores brasileiros e no mercado internacional. Revista Ciências Administrativas, 18, 1,13-44.

Jesus, C. de. Jr., Sidonio, L. \& Moraes, V. E. G de. (2011, setembro) Panorama das importações de trigo no Brasil. [Relatório] BNDES Setorial. Recuperado em 14 de março, 2011, de https://web.bndes.gov.br/bib/jspui/bitstream/1408/1602/1/A\%20BS\%20 34\%20Panorama\%20das\%20importa\%C3\%A7\%C3\%B5es\%20de\%20trig o\%20no\%20Brasil_P.pdf

Johansen, S. (1988, june/September) Statistical analysis of cointegrating vectors. Journal of Economic Dynamics and Control, 12, 2/3, 231-254.

Johansen, S. \& Juselius, K. (1990, may) Maximum likelihood estimation and inference on cointegration - with applications to the demand for money. Oxford Bulletin of Economics and Statistics, 52, 2, 169-210.

Ministério da Agricultura Pecuária e Abastecimento. Trigo. 2016. Recuperado em 14 de 2016, de http://www.agricultura.gov.br/vegetal/culturas/trigo

Margarido, M. A., Bueno, C. R. F., Martins, V. A. \& Tomaz, I. F. (2007) Análise dos efeitos preço e câmbio sobre os preços da farinha de trigo na cidade de são paulo: uma aplicação de modelos de séries de tempo. Pesquisa \& Debate, 18, 2 (32), 251-281.

Margarido, M. A., Kato, H. T. \& Ueno, L. H. (1994) Análise da transmissão e preços no mercado de tomate no estado de São Paulo. Agricultura em São Paulo, 41, 3, 135-159.

Margarido, M. A., Turolla, F. A. \& Bueno, C. R. F. (2014) Análise da volatilidade e transmissão de preços entre os mercados internacionais de petróleo e soja. Organizações Rurais \& Agroindustriais, 16, 1, 123-138.

Margarido, M. A., Turolla, F. A. \& Fernandes, J. M. (2001) Análise da elasticidade de transmissão de preços no mercado internacional de soja. Pesquisa \& Debate, 12, 2(20),5-40.

Mundlak, Y. \& Larson, D. F. (1992) On the transmission of world agricultural prices. World Bank Economic Review, 6, 3, 399-422.

Nogueira, F. T. P., Aguiar, D. R. D. \& Lima, J. E. de. (2005, maio/agosto) Integração espacial no mercado brasileiro de café arábica. Nova Economia, $15,2,91-112$.

Santos, E. dos. \& Wolff, L.; Souza, A. M. (2013, março) Transmissão e a influência do volume dos estoques públicos sobre o preço do arroz no Brasil. Ciência Rural, 43, 3, 559-564. 
Silva, F. M. da \& Machado, T. de A. (2009) Transmissão de preços da soja entre o Brasil e os estados unidos no período de 1997 a 2007. Revista Economia e Desenvolvimento, 21, 85-103.

Silva, J. J. da C. \& Lages, A. M. G. (2011, dezembro) Comercialização de produtos agropecuários em alagoas: um estudo transmissão de preços. Revista Nexos, 5, 9, 157-176.

Silva, W. V. da, Santo, E. L. \& Silva, L. S. C. V. da. (2003, julho/setembro) Cointegração entre os preços da soja cotados nos mercados brasileiro e norteamericano: uma análise empírica. Caderno de Pesquisas em Administração, $10,3,69-78$.

Tonin, J. M. \& Barczsz, S. S. (2007, julho) Transmissão de preços da soja entre os mercados externo e interno: uma abordagem para a região de Maringá. A Economia em Revista, 15, 1, 35-46.

United States Department of Agriculture. 2016. Economic Research Service: USDA Wheat Baseline, 2015-24. Recuperado em 11 de abril, 2016, de http://www.ers.usda.gov/topics/crops/wheat/usda-wheat-baseline,-201524.aspx

United States Department of Agriculture. 2013. Economic Research Service:ing Countries Dominate World Demand for Agricultural Products.Recuperado em 11 de abril, 2016, de http://www.ers.usda.gov/amber-waves/2013august/developing-countries-dominate-world-demand-for-agriculturalproducts.aspx\#.VxFTIUwrLIX

Yang, J., Zhang, J. \& Leatham, D. J. (2003) Price and volatility transmission in international wheat futures markets. Annals of Economics and Finance, 4, $1,37-50$. 